\title{
Management of a Refractory EIF3E-RSP02 Fusion Positive Pilomatrical Cancer Patient with Encyclopedic Tumor Analysis Guided Treatment
}

\section{Revati Patil, Pradip Devhare, Ajay Srinivasan, Dadasaheb Akolkar*, Darshana Patil, Vineet Datta, Ashwini Ghaisas, Chirantan Bose, Shankar Somashekar, Jinumary John, Sanket Patil, Sachin Apurwa, Navin Srivastava and Rajan Datar}

Datar Cancer Genetics Limited, Nasik, India

*Corresponding Author: Dadasaheb Akolkar, Research and Innovation Department,

Datar Cancer Genetics Limited, Nasik, India.
Received: July 21, 2020

Published: September 25, 2020

(C) All rights are reserved by Dadasaheb

Akolkar., et al.

\begin{abstract}
Pilomatrical carcinoma is an exceedingly rare, locally aggressive malignancy of the hair follicle. It may arise de novo as a solitary lesion or through malignant transformation from its pre-existing benign counterpart, pilomatrixoma. There are no standard treatment guidelines and wide surgical excision of the primary lesion followed by adjuvant radiotherapy remains principal treatment modality. However, high recurrence rate after primary treatment, presence of nodal and systemic metastases which is non-responsive to chemotherapy are major concerns in disease management. Here, we report a case of a 45-year-old female with recurrent pilomatrical carcinoma who opted for personalized treatment guidance based on Encyclopedic Tumor Analysis (ETA). Molecular analysis of tumor tissue identified a novel EIF3E-RSPO2 gene rearrangement, SETD2 mutation and overexpression of VEGFA and RET genes. These molecular alterations along with synchronous chemo-sensitivity assay on tumor derived cells helped in defining combinatorial off label therapy regimens which countered disease progression at each instance and led to the durable regression of primary as well as metastatic lesions. This personalized treatment management strategy described herein effectively addressed advanced, refractory disease for which current Standard of Care (SoC) options were unviable.
\end{abstract}

Keywords: Pilomatrical Cancer; EIF3E-RSP02; Personalized Treatment; VEGFA; Bevacizumab; Wnt Signaling; SETD2; Everolimus

\section{Abbreviations}

ETA: Encyclopedic Tumor Analysis; SoC: Standard of Care; IHC: Immunohistochemistry; MAF: Mutant Allele Fraction; PFS: Progression-Free Survival; OS: Overall Survival

\section{Introduction}

Pilomatrixoma, also called as calcifying epithelioma of Malherbe is a relatively common benign cutaneous adnexal tumor derived from hair matrix cells, occurring usually during first two decades of life [1,2]. The locally aggressive malignant counterpart of pilomatrixoma, i.e. 'pilomatrical carcinoma' or 'malignant pilomatrixoma' $[3,4]$ is an extremely rare tumor seen in adults with strong tendency to relapse locally, even after excision with tumorfree surgical margins $[5,6]$. Though the majority of pilomatrical carcinomas develop de novo, malignant transformation from a preexisting pilomatrixoma has been reported [6,7]. Pilomatrical carcinomas mostly occur in the head and neck, upper extremities and buttocks [1-8]. Although, it was considered to be a low-grade malignant tumor, its significant metastatic potential is being evident $[1,4,5,7]$. There are presently no standard of care (SoC) systemic treatments for pilomatrical carcinoma and common treatment approaches include surgical resection as well as physician's choice of systemic treatments, including off label cytotoxic and targeted agents. However, selection of such agents without any evidence of 
efficacy may be associated with sub-optimal treatment responses and poor outcomes, as is generally observed in case of pilomatrical carcinoma. On the other hand comprehensive multi-analyte interrogation of the tumor can reveal latent actionable vulnerabilities for selection of appropriate antineoplastic agents with a projected efficacy. We have previously described the utility of ETA (Encyclopedic Tumor Analysis) which includes immunohistochemistry (IHC), immunocytochemistry (ICC), NGS evaluation of mutations in cell free DNA (cfDNA), tumor transcriptome (mRNA) analysis and in vitro chemosensitivity profiling of viable tumor cells $[9,10]$. In the present report, we describe a case of metastatic Pilomatrical carcinoma wherein ETA guided personalized therapeutic interventions helped disease management when tumor became refractory on all standard measures including surgical intervention, radiotherapy and systemic therapy.

\section{Case Report}

The case described in this manuscript is a retrospective observational report of a single patient who opted to receive personalized cancer treatment based on Encyclopedic tumor analysis (ETA) approach [9]. The process of ETA and related methodologies have been explained previously $[9,10]$. In vitro chemosensitivity profiling of viable circulating tumor associated cells (CTACs) from peripheral blood was performed as described previously [11].

A 43-year-old female was referred to a tertiary regional cancer care center in April 2014 for recurrent polypoidal scalp lesion with cervical lymphadenopathy. As a primary treatment, patient underwent wide excision surgery of scalp lesion in February 2013. After one year, disease recurrence was managed again by wide excision of scalp lesion with rotation flap and modified neck dissection in April 2014. Histopathological examination (HPE) revealed malignant tumor composed of nests and cords of basaloid cells and ghost or shadow cells. She received radiotherapy (30 cycles of $60 \mathrm{~Gy}$ ) along with five cycles of weekly Cisplatin in June 2014. After three years (July 2017), there was an appearance of rounded isoechoic lesion in left supraclavicular region. HPE of left supraclavicular biopsy confirmed metastatic pilomatrical carcinoma, for which she received three cycles of systemic Paclitaxel + Carboplatin followed by eight cycles of weekly Paclitaxel + Cisplatin till January 2018. After six months, follow up PET-CT scan revealed metastatic lesions in bilateral lung parenchyma, left pleura, mediastinal lymph nodes, thyroid and left upper arm skeletal muscle. These episodes represented failure of disease management by all aforementioned primary treatment modalities. Patient opted for Encyclopedic Tumor Analysis (ETA) to receive personalized treatment guidance to manage her metastatic, refractory disease (Figure $1 \mathrm{~A}$ ).

ETA of tumor tissue revealed SETD2 p.Q2139* (MAF 77.7\%), EIF3E (Exon1) - RSPO2 (Exon 2) gene rearrangement (457 reads) as well as overexpression of VEGFA (6.4 fold), RET (4.8 fold) and SRC (2.2 fold) (Table 1). Cytotoxic drugs Etoposide and 5-Fluorouracil showed maximum sensitivity as determined by in vitro chemo-sensitivity analysis using viable tumor cells obtained from fresh tissue biopsy. Based on molecular and chemo-sensitivity analyses, patient was advised Bevacizumab (500 mg IV) (VEGFA overexpression) and 5-fluorouracil (850 mg IV) with Etoposide (50 mg) (chemo-sensitivity), respectively in July 2018. In addition, Quercetin, Celecoxib, Atorvastatin and Doxycycline were also advised for inhibition of activated WNT, MAPK and MMP pathways detected in tissue transcriptome analysis. Follow-up PET-CT scan in September 2018, revealed partial response (PR) to treatment in the form of reduction in size and activity of pleural deposits, mediastinal lymph nodes and skeletal muscle deposits in left upper arm (Figure 1B and 1C). Patient showed PR till December 2018 and continued on same regimen. Serum levels of tumor markers (CEA, LDH, serum albumin) were within normal range, except for mildly elevated C-Reactive Protein (CRP). Patient presented with mild (Grade 1 - 2) adverse effects such as anorexia, fatigue, myalgia and pyrexia. Follow-up PET-CT scan in May 2019 showed increase in size of known recurrent lesions in the scalp. However, patient defaulted from treatment until October 2019, where clear disease progression was observed in the form of increase in size of recurrent primary and all known metastatic deposits. Patient was unwilling for a biopsy. Hence $15 \mathrm{~mL}$ peripheral blood was collected in EDTA vacutainers and used for ETA. There were no targetable gene variations or fusions detectable by NGS. However, ICC profiling of Circulating Tumor Cells CTCs was indicative of mTOR activation, while in vitro chemosensitivity profiling of viable circulating tumor associated cells (CTACs) from peripheral blood showed sensitivity to Cyclophosphamide and Docetaxel. Based on these findings, regimen of Cyclophosphamide (950 mg), Docetaxel (60 mg) along with Everolimus (5 mg, 1 OD) was recommended. Patient received this regimen from November 2019 to February 2020. Follow-up PETCT scan in January 2020 showed PR as evidenced by regression of all primary and metastatic deposits. However, patient defaulted from treatment in February 2020 and was subsequently lost to follow-up. 


\begin{tabular}{|c|c|c|c|c|}
\hline No. & Analysis & Gene & MAF/Fold/Reads & Method \\
\hline ETA-1 & Mutation & SETD2 p.Q2139* & MAF 77.7\% & OCAV3 \\
\hline \multicolumn{5}{|l|}{ Jul-18 } \\
\hline & Gene Fusion & EIF3E (Exon1) - RSPO2 (Exon 2) & 457 reads & OCAV3 \\
\hline & Gene Expression & VEGFA & 6.4 fold & Transcriptome \\
\hline & & RET & 4.8 fold & \\
\hline & & MAPK7 & 3 fold & \\
\hline & & MAPK12 & 3.9 fold & \\
\hline & & SRC & 2.2 fold & \\
\hline & & MMP9 & 3.4 fold & \\
\hline & & MMP11 & 3.3 fold & \\
\hline & & MMP15 & 3.4 fold & \\
\hline & & MMP16 & 5.1 fold & \\
\hline & Immunohistochemistry & AR & Negative & IHC \\
\hline & In vitro Drug response & 5-Fluorouracil + Etoposide & NA & Chemo-sensitivity \\
\hline \multicolumn{5}{|l|}{ ETA-2 } \\
\hline \multirow[t]{2}{*}{ Oct-19 } & In vitro Drug response & Cyclophosphamide + Docetaxel & NA & Chemo-sensitivity \\
\hline & Immunocytochemistry & mTOR expression & NA & CTC-ICC \\
\hline
\end{tabular}

Table 1: Encyclopedic tumor analysis (ETA) findings.

OCAV3: Oncomine Comprehensive Assay v3; MAF: Mutant Allele Frequency; Fold - Upregulation of target gene compared to expression in normal skin RNA; MMP: Matrix Metalloproteinases; ICC: Immunocytochemistry.

$\mathbf{A}$
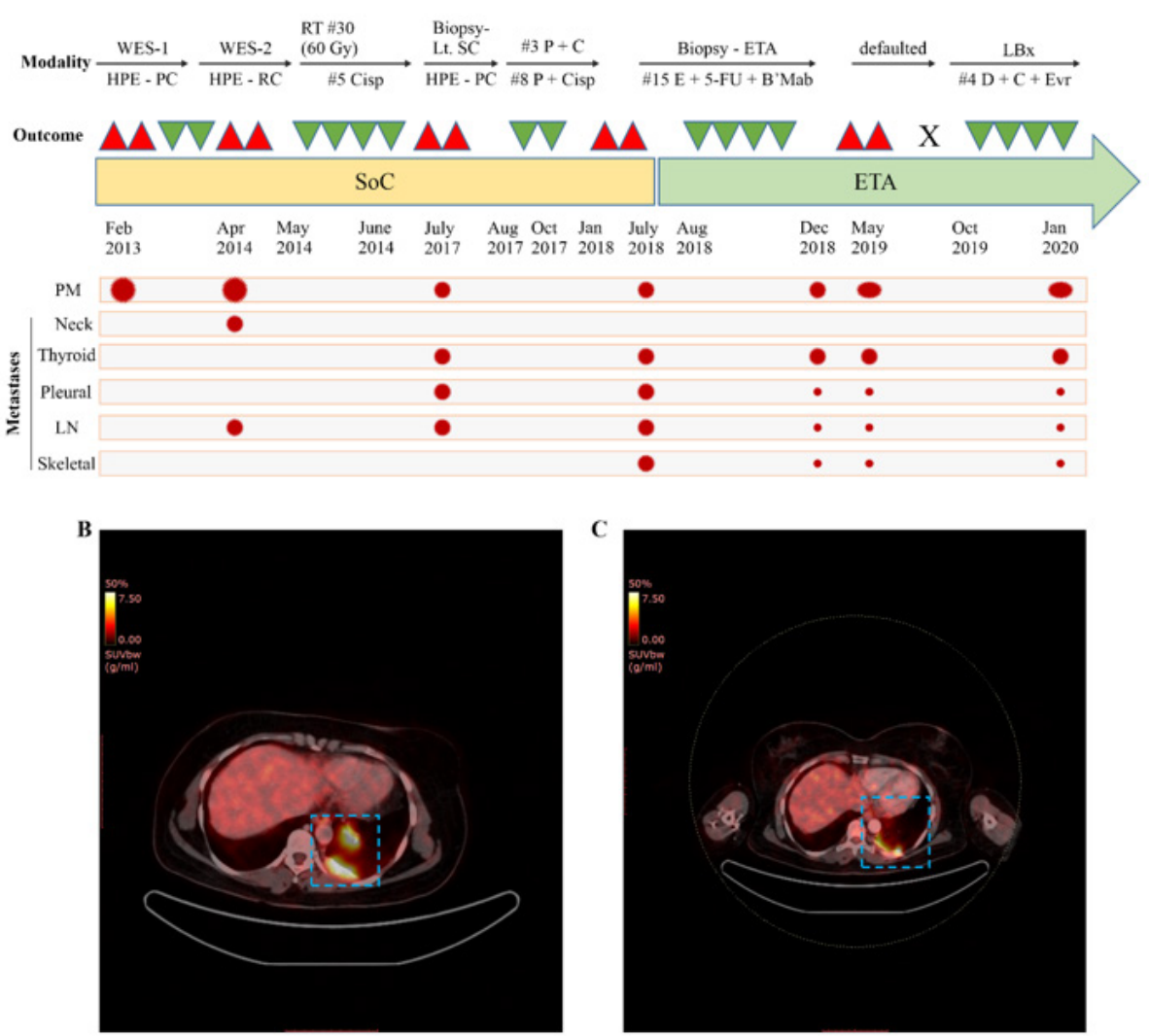

Figure 2: (A) Timeline of events for disease management using Standard of Care (SoC) and Encyclopedic Tumor Analysis (ETA) guided treatment modalities. WES: Wide excision of scalp; HPE: Histopathological examination; PC: Pilomatrical carcinoma; RC: Recurrence; RT: Radiotherapy; Lt.SC: left supraclavicular mass; Cisp: Cisplatin; E + 5-FU + B'Mab: Etoposide + 5-fluorouracil + Bevacizumab; LBx: liquid biopsy; X: defaulted for follow-up; D + C + Evr: Docetaxel + Cyclophosphamide + Everolimus; $\triangle$ : progression/recurrence; $\nabla$ : treatment response/regression; presence of malignant mass at various sites; PM - primary scalp lesion, LN: lymph node. D Disease response in indicated follow ups represents reduction in size of malignant mass. (B-C) Encycolopedic Tumor Analysis (ETA) guided Treatment response was assessed by 18F-fluorodeoxyglucose (FDG)-positron emission tomography-computed tomography (PET-CT) indicating significant regression of left pleural mass (C) compared to baseline scan (B). 


\section{Discussion and Conclusion}

Pathogenesis of pilomatrical carcinoma is unclear, whether it is a malignant transformation of pilomatrixoma or arises de novo. However, mutations in the CTNNB1 gene, which occur in both, points towards a possible common pathogenesis [5,6]. Though Pilomatrical carcinoma usually behaves like a low-grade malignancy, it has a high propensity for local recurrence. It has been reported that, approximately half of the cases of pilomatrical carcinoma were relapsed $[4,12]$. This tumor also displays metastatic behavior which is reported to be associated with local tumor recurrence [6], with metastatic rate ranging between $13 \%$ and $16 \%$. The most common site of metastases are regional lymph nodes, followed by lung, bone, brain, and other viscera $[6,13]$.

The treatment of choice for this locally aggressive tumor is surgical excision with adequate margins. Adjuvant radiotherapy may be started after excision. In case of extensive local infiltration or presence of distant metastasis, chemotherapy is also necessary though with low success rate $[1,3]$. Present case had previously progressed on all primary treatment modalities with disease recurrence as well as metastases. In absence of SoC options and failure of physician's choice of treatments, the patient opted for ETA which identifies molecular and functional vulnerabilities of the multi-layered tumor interactome which in turn facilitates selection of efficacious treatment options for patients with $\mathrm{r} / \mathrm{r}$ m-cancers [9]. ETA approach was instrumental in identifying combination regimens which achieved tandem targeting of tumor vulnerabilities and yielded significant treatment response even in this heavily pretreated patient.

Tumor in the present case represented some characteristic molecular signatures like EIF3E (Eukaryotic Translation Initiation Factor 3 Subunit E) Exon 1 - RSPO2 (R-spondin family gene) Exon 2 gene rearrangement. This fusion transcript is expected to produce a functional RSPO2 protein driven by the EIF3E promoter which is potentially involved in canonical Wnt signaling in colon cancer [14]. These fusions are known to occur exclusively in tumors that do not contain APC or CTNNB1 gene mutations [14]. Pilomatrical carcinoma and its benign counterpart Pilomatricoma are known to harbor CTNNB1 gene mutations [5] and identification of R-spondin gene arrangement in present case revealed another unidentified potential mechanism of Wnt signaling in pathogenesis of Pilomatrical carcinoma. This also allowed us to recommend potentiating drugs Quercetin and Celecoxib in treatment regimen [15].

Additionally, SETD2 p.Q2139* mutation was identified in tumor tissue at high mutation frequency. SETD2 is an epigenetic tumor suppressor known to have histone methyltransferase activity and its inactivation was reported to be associated with tumorigenesis by different mechanisms [16]. Inactivation of SETD2 reported to confer cisplatin chemo resistance in non-small cell lung cancer cells by regulating ERK and Wnt/ $\beta$-catenin signaling in Osteosarcoma [16]. This implicates the possibility of chemo resistance in present case who received Cisplatin based regimens in her prior treatment modalities. Overexpression of VEGFA along with other tyrosine kinases RET and Src suggested activation of proangiogenic signaling. Bevacizumab was included as a targeting agent to target angiogenic signaling along with cytotoxic drugs during first ETA approach (July 2018). Partial response was evaluated with this regimen until December 2018 (Figure 1C). At subsequent recurrence (post-defaulting) further recommendations were based on liquid biopsy approach which also led to regression of tumor. Notably, in both instances, ETA guided treatments were not only efficacious but also well tolerated with no grade 3 or higher treatment related adverse events (AEs). In the present case of recurrent, refractory metastatic disease, ETA-guided treatment achieved an overall survival (OS) of 1.8 years (censored up to April 2020). However durable response could not be achieved at both instances owing to patient's inability to comply with therapy. The present case study presents a treatment conundrum that is faced by clinicians routinely, i.e. non-compliance to treatment leading to disease progression/recurrence. In such situations, psychological counselling of patients is of paramount importance to convey the importance of compliance to therapy and to prevent lapses in treatment.

In this case with Pilomatrical carcinoma, ETA guided personalized therapeutic interventions helped disease management where all the standard measures including surgical intervention, radiotherapy and systemic therapy were utilized and tumor became metastatic and refractory. This emphasizes use of precision oncology to improve prognosis and outcomes for patients with such rare type of malignancies lacking standard of care treatment guidelines.

\section{Acknowledgement}

The Authors acknowledge the contribution of HCG-Manavata Cancer Centre and their staff and thank them for the clinical management of the patient. The contribution of Mr. Milind Agnihotri, Dr. Jitendra Karlekar and Mr. Sandip Birari is also acknowledged.

\section{Funding}

This research did not receive any specific grant from funding agencies in the public, commercial, or not-for-profit sectors.

\section{Declarations of Interest}

None. 


\section{Ethics Approval}

The patient provided signed informed consent for the publication of deidentified data and results. Sample collections and therapeutic interventions were carried out at HCG Manavata Cancer Centre, Nasik, India. Cellular and molecular investigations on the patient's samples were carried out at the College of American Pathologists (CAP)-accredited and International Organization for Standardization (ISO)-compliant facilities of Datar Cancer Genetics Limited (DCGL), Nasik, India. All interventional procedures including therapy administration were approved as per standard hospital practices and in concordance with existing ethical, medical and legal requirements.

\section{Statement of Compliance}

All procedures were performed in compliance with relevant laws and institutional guidelines and respective institutional committees have approved them.

With regard to human and animal subjects including, Experiments involving humans: The Code of Ethics of the World Medical Association (Declaration of Helsinki) and experiments involving animals and their associated guidelines are not specifically relevant to this case study.

\section{Bibliography}

1. Abd Elazeem MA and Radi DA. "Pilomatrical carcinoma in the thigh: A case report and review of literature". Journal of Interdisciplinary Histopathology 4.3 (2016): 70-73.

2. Bartoš V and Kullová M. "Pilomatrical carcinoma - a case report and review of the literature". Our Dermatology Online Journal 9.2 (2018): 170-173.

3. Xing L., et al. "Facial pilomatrix carcinomas treated with Mohs micrographic surgery". JAAD Case Reports 4.3 (2018): 253-255.

4. Papadakis M., et al. "Pilomatrix carcinoma: More malignant biological behavior than was considered in the past". Molecular and Clinical Oncology 6.3 (2017): 415-418.

5. Herrmann JL., et al. "Pilomatrix carcinoma: 13 new cases and review of the literature with emphasis on predictors of metastasis". Journal of the American Academy of Dermatology 71 (2014): 38-43.

6. Sassmannshausen J and Chaffins M. "Pilomatrix carcinoma: A report of a case arising from a previously excised pilomatrixoma and a review of the literature". Journal of the American Academy of Dermatology 44.2 (2001): 358-361.
7. Hardisson D., et al. "Pilomatrix carcinoma: a clinicopathologic study of six cases and review of the literature". The American Journal of Dermatopathology 23 (2001): 394-401.

8. Liu CC., et al. "Pilomatrix carcinoma of the head and neck: Case report and literature review". Head and Neck Oncology 6 (2014): 12.

9. Nagarkar R., et al. "Encyclopedic tumor analysis for guiding treatment of advanced, broadly refractory cancers: results from the RESILIENT trial". Oncotarget 10.54 (2019): 56055621.

10. Ranade A., et al. "Adaptive, Iterative, Long-Term Personalized Therapy Management in a Case of Stage IV Refractory NSCLC". Journal of Personalized Medicine 9.3 (2019): 34.

11. Limaye S., et al. "Effect of previous chemotherapy treatments on circulating tumor-associated cells in colorectal cancer". Journal of Clinical Oncology 38.4 (2020): 194-194.

12. Sau P., et al. "Pilomatrix carcinoma". Cancer 71 (1993): 24932498.

13. Melancon JM., et al. "Management of pilomatrix carcinoma: a case report of successful treatment with mohs micrographic surgery and review of the literature". Dermatologic Surgery 37.12 (2011): 1798-1805.

14. Seshagiri S., et al. "Recurrent R-spondin fusions in colon cancer". Nature 488.7413 (2012): 660-664.

15. Sferrazza G., et al. "Nature-derived compounds modulating Wnt/ $\beta$-catenin pathway: a preventive and therapeutic opportunity in neoplastic diseases". Acta Pharmaceutica Sinica $B$ (2020).

16. Chen R., et al. "Histone methyltransferase SETD2: a potential tumor suppressor in solid cancers". Journal of Cancer 11.11 (2020): 3349-3356.

\section{Assets from publication with us}

- Prompt Acknowledgement after receiving the article

- Thorough Double blinded peer review

- Rapid Publication

- Issue of Publication Certificate

- High visibility of your Published work

Website: www.actascientific.com/

Submit Article: www.actascientific.com/submission.php Email us: editor@actascientific.com

Contact us: +919182824667 